\title{
Correction of pixel sensitivity variation and off-axis response for amorphous silicon EPID dosimetry
}

\author{
Peter B. Greer ${ }^{a)}$ \\ Newcastle Mater Hospital, and University of Newcastle, Newcastle, New South Wales, Australia
}

(Received 26 May 2005; revised 3 October 2005; accepted for publication 4 October 2005; published 11 November 2005)

The aim of this work is to determine the pixel sensitivity variation and off-axis dose response of an amorphous silicon electronic portal imaging device (EPID), and develop a correction method to improve EPID dosimetry. The uncorrected or raw pixel response of the aS500 amorphous silicon EPID shows differences in response (sensitivity) of individual pixels as well as a large off-axis differential response with respect to an ion chamber in water. Both can be corrected by division of raw images by the flood-field (FF) image. However, this leads to two problems for dosimetry: (1) the beam profile is present in both the raw image and FF image, and hence is "washed out" of the corrected image, and (2) any mismatch of EPID position between dosimetry and FF calibration means that the beam profile and off-axis response in the raw image and FF are misaligned. This causes artifacts in FF division and dosimetric errors. A method was developed to measure the off-axis response and pixel sensitivity variation separately to allow correction of images at any EPID position while retaining beam profile information. The pixel sensitivity variation is applied to the imager plane and is independent of imager position. The off-axis response depends on the imager plane position relative to the beam central axis. The pixel sensitivities were derived from multiple images of the same symmetric field acquired with the detector displaced laterally between each image. The off-axis response was measured by acquiring off-axis raw images (FF correction removed) and dividing out the off-axis beam fluence and previously determined pixel sensitivity differences. The dosimetric errors due to lateral and vertical detector displacement with the conventional FF calibration method were measured and compared to the new method. Corrected EPID profiles were then compared to beam profiles measured with ion chamber in water for open fields. The EPID was found to have a large off-axis differential response with respect to an ion chamber in water, particularly for $6 \mathrm{MV}$. This increased to $13 \%$ at $15 \mathrm{~cm}$ off-axis for $6 \mathrm{MV}$, and $3.5 \%$ for $18 \mathrm{MV}$ at the isocenter plane. The dosimetric errors introduced by detector displacement with conventional FF calibration were found to be approximately $1 \%$ per centimeter of lateral detector displacement and $0.1 \%$ per centimeter of vertical displacement. These were reduced to less than $1 \%$ for any position with the new correction method. Corrected EPID images agreed with ionchamber measurements to within $2 \%$ (excluding penumbra and low-dose areas outside the field) for various field sizes. The new correction method gives consistent dosimetry for any EPID position and retains beam profile information in the image. (C) 2005 American Association of Physicists in Medicine. [DOI: 10.1118/1.2128498]

Key words: amorphous silicon, dose verification, EPID

\section{INTRODUCTION}

The use of amorphous silicon electronic portal imaging devices (EPIDs) for dosimetric applications such as accelerator quality assurance, in vivo dosimetry, and verification of intensity modulated radiation therapy (IMRT) is of current interest. They require little experimental setup time and provide high-resolution, two-dimensional measurements. However, there are many challenging problems that must be resolved in order to perform accurate dosimetry with these devices. For the aS500 EPID (Varian, Palo Alto, CA) the dose response has been found to be linear. ${ }^{1-4}$ However, the change in EPID signal with field size is different from that of a water phantom. ${ }^{4-6}$ To account for this, several investigators have measured or modeled the scatter kernel and glare kernel of the EPID..$^{1,3,7,8}$ The EPID has also been shown to over- respond to low-energy photons by McCurdy et al., ${ }^{3}$ who investigated the central-axis response of the EPID to varying photon energy. They found that the EPID over-responds to low-energy photons due to the photoelectric effect in the high-atomic number phosphor layer. Backscatter from components of the EPID support arm downstream from the detector have also been found to influence the signal by up to $5 \%{ }^{9}$ Saturation of the detector can occur for higher dose rates, and EPID positions close to the isocenter. ${ }^{6}$ Sampling problems can also confound measurements, as the EPID exhibits a dead time in acquisition every 64 frames as the image buffer is cleared. ${ }^{4}$ The manufacturer has partially resolved this issue; however, some detector saturation can now occur due to a longer sampling time for the 65 th frame. ${ }^{6}$

Differences in pixel response must also be accounted for, as the uncorrected or raw pixel response of the aS500 shows 
differences in response (sensitivity) of individual pixels. There is also an off-axis differential (energy) response with respect to an ion chamber in water. ${ }^{10}$ Both the pixel sensitivity variation and off-axis differential energy response can be corrected by division of raw images by the flood-field (FF) calibration image. This FF image is acquired with a large open field, and all raw EPID images are divided by it before they are stored and displayed. However, the beam profile is present in both the raw image and FF image, and is therefore washed out of the final stored image. To restore the beam profile the EPID images can be multiplied by a beam profile matrix. The off-axis differential energy response of the EPID has received little attention to date. Siebers et al. ${ }^{11}$ developed a Monte Carlo (MC) method to predict the image recorded by the EPID for open and IMRT fields. This model includes beam spectral changes off-axis, and they reported excellent agreement between MC prediction and measured EPID images for open fields. This requires that the FF calibration image does not include beam profile or off-axis spectral effects. They acquired the FF with a nearly uniform flat beam using buildup and a large source-to-detector distance. The MC calculated images were also multiplied by a correction matrix, representing the ratio of the EPID measurement and the MC result for a large open field. This corrected the MC image to the field flatness measured in the FF-corrected EPID image.

As the beam profile is present in the FF image and the acquired EPID dosimetry image, then any displacement of the detector will result in misalignment of these for FF division. The FF-corrected EPID image will no longer be flat, and multiplication of the image by a profile function will not accurately restore the beam profile. Moreover, the off-axis differential energy response of the EPID will depend on the distance of the pixel from the central axis. If the EPID is displaced relative to the central axis between FF calibration and dosimetry, then the off-axis differential energy response in the two images will also be misaligned. The EPID positioning arm is not rigid and differences in positioning occur from day to day. As the gantry is rotated the EPID arm sags and differences in position laterally/longitudinal of several centimeters can occur. Differences in position vertically of up to $4 \mathrm{~cm}$ can also occur between gantry 0 to $180 \mathrm{deg}$ positions. The EPID can also be positioned at different distances from the source $(105-180 \mathrm{~cm})$, and at various lateral and longitudinal positions for clinical imaging or for dosimetry of larger asymmetric fields.

In this work the pixel sensitivity variation and off-axis differential energy response of the EPID are experimentally investigated. A method is developed to measure the off-axis differential energy response and pixel sensitivities separately. This allows correction for any EPID position while retaining beam profile information in the image. The EPID matrix is corrected for pixel sensitivities regardless of its position in the beam, while the off-axis differential energy response correction is first centered on the beam central axis, and then applied to the matrix, taking into account its current position. The effect of imager displacement between conventional FF calibration and dosimetry measurement is examined to determine the magnitude of the dosimetry errors that are introduced. The new correction method is then applied to these images to determine the improvement in dosimetric accuracy. EPID profiles corrected for pixel sensitivity and offaxis differential energy response are then compared to beam profiles measured with an ion chamber in water at $d_{\max }$ for open fields.

\section{METHODS AND MATERIALS}

\section{A. EPID}

The aS500 EPID (Varian, Palo Alto, CA) detection layers consist of a $1 \mathrm{~mm}$ thick copper plate, a $134 \mathrm{mg} / \mathrm{cm}^{2}$ gadolinium oxysulfide phosphor layer, and a $40 \times 30 \mathrm{~cm}^{2}$ amorphous silicon light detector matrix $(512 \times 384$ pixels $)$. Further details of the EPID structure can be found in the literature. ${ }^{11}$

All measurements were performed on an aS500 amorphous silicon EPID and a 21EX accelerator (Varian) with nominal 6- and $18 \mathrm{MV}$ energy photon beams. The dose rate was $300 \mathrm{MU} / \mathrm{min}$ for all measurements. Unless otherwise stated, the EPID was positioned with the surface of the detector at $105 \mathrm{~cm}$ from the source, with $0.5 \mathrm{~cm}$ of added Perspex buildup for $6 \mathrm{MV}$, and $2.0 \mathrm{~cm}$ for $18 \mathrm{MV}$. The buildup was placed on top of the EPID plastic collision cover and completely encompassed the detector. While added buildup may not be required for accurate EPID dosimetry, $d_{\max }$ buildup was used in this work to enable comparison with ion-chamber measurements in water recorded at this depth. The extent to which this work can be applied to the detector used without any buildup is examined in Sec. II. The EPID was operated with continuous frame acquisition during beam delivery. The number of reset frames before image acquisition was zero. The reset frame every 64 frames was removed by a software update. The PV CLIENT software version was 6.1.13 with IAS2 software version 6.1.11, and the detector IDU11 model.

\section{B. Conventional flood-field calibration}

To account for individual pixel background signals, a dark-field (DF) image is acquired without radiation. Traditionally, to determine the individual pixel sensitivities or gains, the EPID is irradiated with an open "uniform" field and the resulting raw pixel value image (DF-corrected) is known as the flood field (FF). When a raw image is acquired by the EPID, the DF is subtracted and the image is then divided by the normalized FF image

$$
I(i, j)=\left(\frac{I_{\text {raw }}(i, j)-\mathrm{DF}(i, j)}{\mathrm{FF}(i, j)}\right) \mathrm{FF}_{\text {mean }} .
$$

The EPID software reports the postprocessed FF-corrected image $I . \mathrm{FF}_{\text {mean }}$ is the mean value of the FF image.

\section{Raw EPID response}

The FF or raw pixel response of the EPID to a 40 $\times 30 \mathrm{~cm}^{2}$ open field with $d_{\max }$ buildup was compared to ion- 
chamber profiles measured in water. Dose profiles at $d_{\max }$ were measured with an IC15 $0.125 \mathrm{cc}$ ion chamber (Wellhofer, Schwarzenbruch, Germany) in a water tank. The depths of ion-chamber measurements were 1.5 for $6 \mathrm{MV}$, and $3.0 \mathrm{~cm}$ for $18 \mathrm{MV}$, with a source-to-surface distance (SSD) of $100 \mathrm{~cm}$.

\section{Pixel sensitivity variation}

The pixel sensitivity map (PSM) is defined here as the EPID pixel response to a beam that has uniform intensity and the same energy spectrum at all pixel locations in the detector matrix. This function contains only the inherent differences in response or gain of the individual pixels in the imaging matrix, specified relative to the central pixel. To measure the pixel sensitivity variation, the EPID was irradiated with a symmetric $10 \times 25 \mathrm{~cm}^{2}(10 \mathrm{~cm}$ in the cross-plane direction) $6 \mathrm{MV}$ field, with the EPID detector displaced $2.5 \mathrm{~cm}$ laterally in the cross-plane direction between each irradiation. Six EPID offsets were used in each direction up to $15 \mathrm{~cm}$. The field width of $10 \mathrm{~cm}$ was chosen to achieve a relatively uniform beam at the center of the field without the effect of penumbral falloff. A larger field would have limited the lateral extent to which the pixel sensitivity variation could be measured due to irradiation of the electronics at the edge of the detector. The detector can move laterally up to $15 \mathrm{~cm}$; therefore, a $10 \mathrm{~cm}$ field width enables data to be measured out to this distance, with $5 \mathrm{~cm}$ at the detector edge necessarily missed. The $25 \mathrm{~cm}$ dimension was arbitrarily chosen to cover nearly the entire detector; however, a smaller field could have been utilized. Each irradiation was $150 \mathrm{MU}$, with the integrated pixel response obtained by multiplication of the pixel values by the number of frames acquired. As the EPID reports the FF-corrected pixel values, the raw values were first obtained by multiplication of the FF-corrected EPID image by $\mathrm{FF} / \mathrm{FF}_{\text {mean }}$. The DF correction was retained, as this removes offset signals of individual pixels and is recorded without radiation present. A composite pixel sensitivity variation along the central axis in the cross-plane direction was formed. This was obtained by combining the central $2.5 \mathrm{~cm}$ (16 pixels) of the one-dimensional cross-plane profile from each raw EPID image. This central region of the field approximates a uniform intensity beam without variation in the energy spectrum. This was repeated for $18 \mathrm{MV}$ photons. The use of the same $10 \times 25 \mathrm{~cm}^{2}$ field for each irradiation also ensured that there was no change in scatter conditions for the measurements. The detector backscatter is unlikely to depend on cross-plane position, as the support arm components are relatively uniform in this direction. ${ }^{9}$

The measurements outlined above derive the pixel sensitivity variation for a central-axis profile in the cross-plane direction. To derive the two-dimensional PSM, the ratio of the cross-plane central-axis profile of the conventional open $\left(40 \times 30 \mathrm{~cm}^{2}\right) \mathrm{FF}$ to the cross-plane central-axis profile of the pixel sensitivity variation was determined by division. This ratio includes the dose profile for the large open field in the cross-plane direction along the central axis, as well as the off-axis response of the EPID. By assuming the dose profile and off-axis response are radially symmetric, the twodimensional PSM can be obtained by dividing the conventional open-field FF by the radially extended ratio function.

Before the one-dimensional ratio function was radially extended, noise and discontinuities were reduced. The discontinuities arose at the junctions of the $2.5 \mathrm{~cm}$ segments of the cross-plane pixel sensitivity profile due to asymmetries in the beam profile in the central region of the $10 \times 25 \mathrm{~cm}^{2}$ fields. To obtain a smooth ratio function the values of the ratio function at $2.5 \mathrm{~cm}$ intervals, corresponding to the center of each of the $10 \times 25 \mathrm{~cm}^{2}$ fields, were sampled and a smooth spline fitted to these. This function was slightly asymmetric about the central axis due to asymmetry in the large-field beam profile. This asymmetry is present in the conventional FF, but not in the pixel sensitivity. The ratio function was folded about the central axis, as this asymmetry had to be removed before the function was radially extended. This folding introduces spurious asymmetry into the PSM; however, this is small. The folded ratio function values were found to be within $0.5 \%$ of the unfolded values. As the crossplane pixel sensitivity profile (and hence ratio function) was only measured to $\pm 15 \mathrm{~cm}$, the ratio function was linearly extrapolated to $\pm 25 \mathrm{~cm}$. This was then radially mapped to a $40 \times 30$ matrix with the same resolution as the EPID pixel matrix $(512 \times 384)$. The two-dimensional PSM was then derived by division of the conventional open-field FF by this two-dimensional matrix.

The pixel sensitivities will not depend on whether extra buildup is used on the EPID. However, the open FF EPID profile may differ between the bare imager and the imager with $d_{\max }$ buildup. This would result in a difference in the ratio function to obtain the PSM from the conventional FF. Ideally, the ratio function would be measured for the buildup conditions that will be used for EPID dosimetry. To examine this, the difference between the open-field EPID profile for the bare imager and $d_{\max }$ buildup was determined for 6 and $18 \mathrm{MV}$, for a $40 \times 30 \mathrm{~cm}^{2}$ field.

Another method that has been applied to FF measurement has been to use sufficient buildup so that the incident beam profile is relatively flat. ${ }^{4,11,12}$ The buildup is also likely to remove lower energy primary photons and reduce the offaxis response. This method does not account for the off-axis response of the EPID when subsequent dosimetry is performed with $d_{\max }$ buildup. The pixel sensitivity variation measured as described above was compared to conventional FF calibration with a large, relatively uniform input beam formed by placing $8 \mathrm{~cm}$ of Perspex buildup on the EPID. The EPID was positioned at $150 \mathrm{~cm}$ from the source with a large open $28 \times 21 \mathrm{~cm}^{2}$ field. The Perspex was supported just above the detector with a gap of approximately $3 \mathrm{~cm}$ to the detector surface.

\section{E. Off-axis response}

Having determined the pixel sensitivity variation of the EPID, it is now possible to determine the off-axis differential energy response. The EPID detector position was fixed about the central axis and the $10 \times 25 \mathrm{~cm}^{2}$ field was moved asym- 

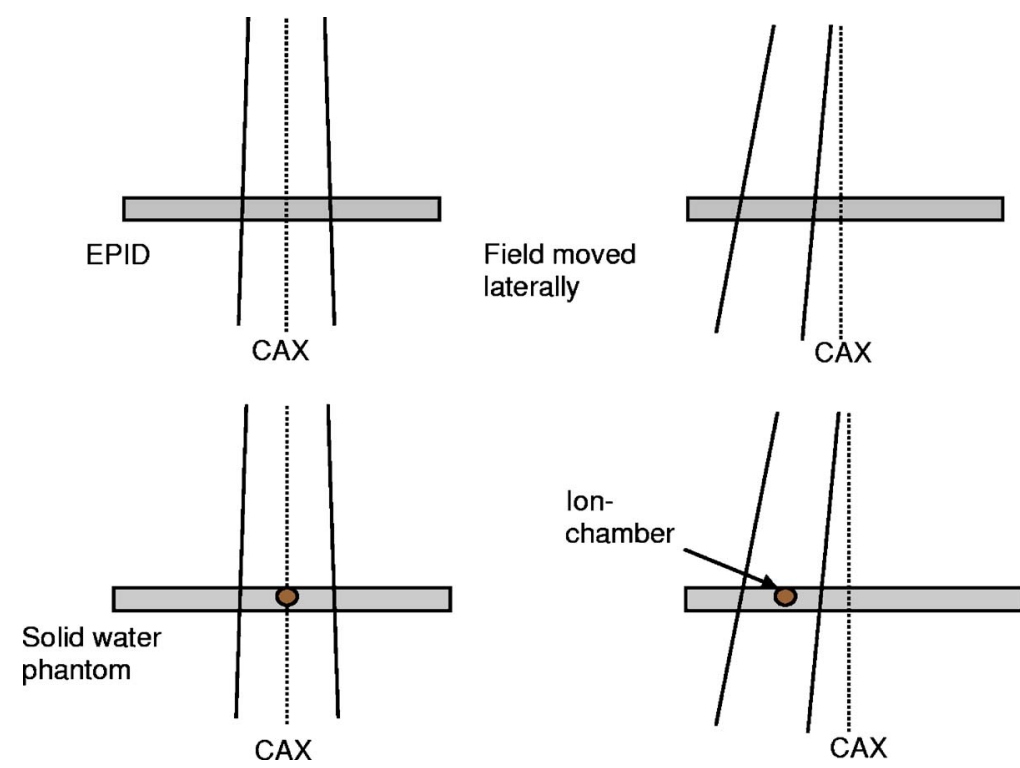

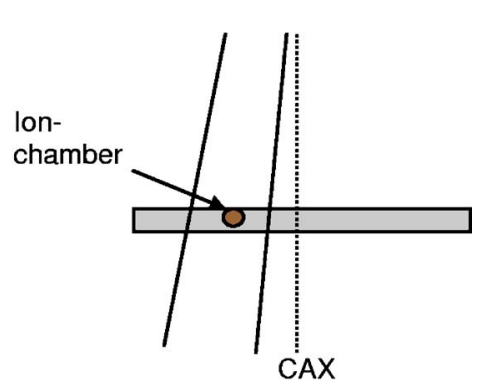

FIG. 1. Illustration of method to measure the off-axis differential energy response of the EPID. The detector position was fixed and the field was moved asymmetrically in $2.5 \mathrm{~cm}$ increments to irradiate the EPID. The central $2.5 \mathrm{~cm}$ of each profile in the cross-plane direction was combined to determine the off-axis EPID response. The dose was measured for these fields with an ion chamber in solid water to estimate the beam relative fluence. Removal of the beam fluence and pixel sensitivity yields the off-axis differential response of the EPID with respect to an ion chamber in water. metrically by $2.5 \mathrm{~cm}$ in the cross-plane direction between each irradiation (Fig. 1). A composite cross-plane EPID profile was formed as before from the central $2.5 \mathrm{~cm}$ of each image. This profile contains EPID pixel variation, off-axis beam fluence, and the off-axis differential energy response of the EPID.

To determine the off-axis beam fluence, ion-chamber measurements were made in a solid water phantom. The phantom was $30 \times 30 \mathrm{~cm}^{2}$ and $8 \mathrm{~cm}$ thick. The results should not depend on the thickness of the phantom, as the backscatter for each asymmetric field of the same size should be very similar. The ion chamber was positioned at $d_{\max }(1.5 \mathrm{~cm}$ for $6 \mathrm{MV}$ and $3.0 \mathrm{~cm}$ for $18 \mathrm{MV}$ ) in the center of each asymmetric field at the same distance as the EPID, and the readings relative to the symmetric field at the central axis obtained (Fig. 1). The pixel sensitivity variation, as determined in the previous section, and beam fluence were then removed from the above EPID profile, yielding the differential response of the EPID with respect to ion chamber in water, with off-axis distance. A similar approach to above was taken, where the off-axis response was smoothed by taking the values at $2.5 \mathrm{~cm}$ intervals corresponding to the center of each of the $10 \times 25 \mathrm{~cm}^{2}$ fields. These values were folded about the central axis to remove any asymmetry. A fourthorder polynomial was then fitted to the data.

A two-dimensional off-axis differential energy response matrix was formed by assuming that this response was radially symmetric, and by mapping the radial function to a Cartesian coordinate system with the same resolution as the EPID pixel matrix. As before, the off-axis differential energy response was only measured to $\pm 15 \mathrm{~cm}$. To allow for detector shift relative to the central axis of $10 \mathrm{~cm}$, the measured response was extrapolated to $\pm 40 \mathrm{~cm}$ using a fourth-order polynomial fit to the measured data, and then mapped to a $60 \times 50 \mathrm{~cm}^{2}(768 \times 640$ pixel $)$ matrix. This means that the off-axis correction can be applied to the entire detector for lateral and longitudinal detector translations of up to $10 \mathrm{~cm}$. Although this extrapolation is large, and will introduce un- certainty into the off-axis differential energy response, the extrapolated correction is likely to be in the region of the beam outside the field. This is because the maximum beam dimension in any direction from the central axis is $\pm 20 \mathrm{~cm}$ (and most beams are smaller), so the off-axis correction beyond this distance will be outside the field edge.

To determine whether the off-axis differential energy response results can be applied to the imager without added buildup, an off-axis image for $12.5 \mathrm{~cm}$ offset from central axis, and a central axis image were acquired with no buildup on the detector. By comparing the ratio of the response at the center of the off-axis image and the central axis with no buildup to the same ratio with $d_{\max }$ buildup, the effect of removing the buildup on the off-axis response results can be estimated.

\section{F. Correction of EPID images}

In the case of IMRT verification where a prediction of the EPID image is made for comparison to the measured EPID image, the PSM correction could be applied to the raw image data, whereas the off-axis response could be applied to the theoretically predicted image. Applying the off-axis response correction to the predicted image would then be independent of imager position. However, as a prediction method was not available to the author at this time, a correction method where both the PSM and off-axis correction are applied to the EPID data was developed.

The pixel sensitivity correction is applied directly to the EPID matrix irrespective of position, while the off-axis response correction is centered on the beam central axis, scaled according to the EPID distance, and then applied to the EPID. The EPID pixel coordinate system is defined as pixel $(0,0)$ at the top left of the imaging matrix. This is the far left of the EPID matrix relative to an observer standing at the end of the treatment couch looking toward the gantry, or the top left of images as they appear on the screen. To correct im- 
ages for the pixel sensitivity variation, the FF correction that is applied during preprocessing by the EPID software is first removed to obtain the raw pixel values $I_{\text {raw }}$

$$
I_{\text {raw }}(i, j)=I(i, j) \frac{\mathrm{FF}(i, j)}{\mathrm{FF}_{\text {mean }}} .
$$

This matrix retains the DF correction. The raw image matrix is then corrected for pixel sensitivity variation by division by the PSM

$$
I^{\prime}(i, j)=\frac{I_{\mathrm{raw}}(i, j)}{\operatorname{PSM}(i, j)} .
$$

The off-axis differential energy response (OAR) must be scaled if the vertical detector position is different from the reference distance $\left(\mathrm{SSD}_{\text {ref }}\right)$ for the off-axis response function.

$$
\mathrm{OAR}_{z}(i, j)=\mathrm{OAR}_{\mathrm{ref}}(i / K, j / K),
$$

with

$$
K=\frac{\mathrm{SDD}_{\mathrm{ref}}}{\mathrm{SDD}_{\mathrm{ref}}+\Delta z} .
$$

The distance $\Delta z$ is the change in distance (positive for increasing distance) from $\mathrm{SSD}_{\text {ref }}$. To account for EPID translation, the OAR is centered on the beam central axis pixel, and then applied to the EPID matrix to give the corrected EPID response

$$
I^{c}(i, j)=\frac{I^{\prime}(i, j)}{\operatorname{OAR}_{z}\left(i+l-p_{x}, j+l-p_{y}\right)},
$$

where the displacement of the EPID relative to the beam central axis in pixels is

$$
\begin{aligned}
& p_{x}=-\Delta x_{E} / \Delta p, \\
& p_{y}=\Delta y_{E} / \Delta p,
\end{aligned}
$$

and $\Delta p$ is the pixel pitch, $\Delta x_{E}$ is the detector lateral displacement $\left(+v_{e}\right.$ is detector movement to the right of an observer standing at the end of the treatment couch, looking toward the gantry), $\Delta y_{E}$ is the detector longitudinal displacement $\left(+v_{e}\right.$ is movement of the EPID toward the gantry). $l$ is the offset of the OAR index, and is due to the fact that this is a larger matrix. It is determined by the increase in matrix size to allow for detector translation. In this case, allowing for translation of $10 \mathrm{~cm}$, the value of $l$ is 127 . For the EPID with no displacement present, the index $(0,0)$ in the EPID matrix corresponds to the same $x$ - and $y$ coordinate as index $(127,127)$ in the extended OAR matrix.

\section{G. Effect of EPID displacement and dosimetry measurements}

The magnitude of dosimetry artifacts introduced by differences in EPID position for the conventional FF-calibration method was investigated. These are due to the misalignment of the beam profile and off-axis response in the raw EPID response and the FF correction. The EPID was FF-calibrated with a $40 \times 30 \mathrm{~cm}^{2}$ open field. An image was then acquired without any detector displacement with the same field size to verify that a completely flat profile was obtained. The detector was then displaced laterally in the cross-plane direction with 5 steps from 0.2 to $3.0 \mathrm{~cm}$ and images acquired at each position. The resulting cross-plane profiles through the central axis in the direction of the displacement were recorded, and the differences from the flat profile without the EPID displaced determined by division. The EPID distance from the source was also increased from 1.0 up to $20.0 \mathrm{~cm}$ below the FF-calibration reference position $(105 \mathrm{~cm})$, and images recorded to determine the magnitude of any dosimetric errors introduced.

The accuracy of the new correction method was then compared to conventional FF calibration with beam profile correction, when detector displacement was present. The profile for the conventional FF method along the central axis in the cross-plane direction was multiplied by a large field $\left(40 \times 40 \mathrm{~cm}^{2}\right) d_{\max }$ water tank profile to "restore" the beam profile to the image. It was first verified that the conventional FF method with beam profile correction matched the EPID profile obtained with the new method when no detector displacement was present. Then, a comparison was made with the detector displaced $3.0 \mathrm{~cm}$ laterally. This was repeated for the detector vertically displaced by $20 \mathrm{~cm}$ between FF calibration $(105 \mathrm{~cm})$ and dosimetry $(125 \mathrm{~cm})$. To compare the profiles to that with no detector displacement, the results were scaled to the isocenter plane.

The accuracy of the EPID correction method was investigated by comparison to profiles measured at $d_{\max }$ with an ion chamber in water. Cross-plane and inplane profiles were measured in a water phantom with the $0.125 \mathrm{cc}$ ion chamber. The source-surface distance was $100.0 \mathrm{~cm}$. Field sizes measured were $5 \times 5,10 \times 10,20 \times 20$, and $40 \times 40 \mathrm{~cm}^{2}$ for both 6 and $18 \mathrm{MV}$. For the $40 \times 40 \mathrm{~cm}^{2}$ fields the tank was offset and a complete profile obtained on one side of the central axis, but with an incomplete profile on the other. Both the EPID and ion-chamber measurements were scaled to $100 \mathrm{~cm}$ from the source for comparison and normalized at the central axis. The EPID images were acquired with $150 \mathrm{MU}$ for each field.

\section{RESULTS}

\section{A. Raw EPID response}

The FF or raw pixel response of the EPID to a 40 $\times 30 \mathrm{~cm}^{2}$ open field with $d_{\max }$ buildup is shown in Fig. 2 . The central-axis pixel value profile in the cross-plane direction is shown for both 6 and $18 \mathrm{MV}$ beams, as well as $d_{\max }$ profiles measured with an ion chamber in a water tank. All profiles have been normalized to the central axis. All profiles have been scaled to the isocenter plane for comparison.

The FF profile recorded by the EPID is higher off-axis than that measured by an ion chamber, particularly for $6 \mathrm{MV}$. This could potentially be due to a gradient effect in the pixel sensitivities, perhaps due to a manufacturing process of the flat-panel matrix. However, the much larger effect for $6 \mathrm{MV}$ suggests that this is due to a response to the beam 


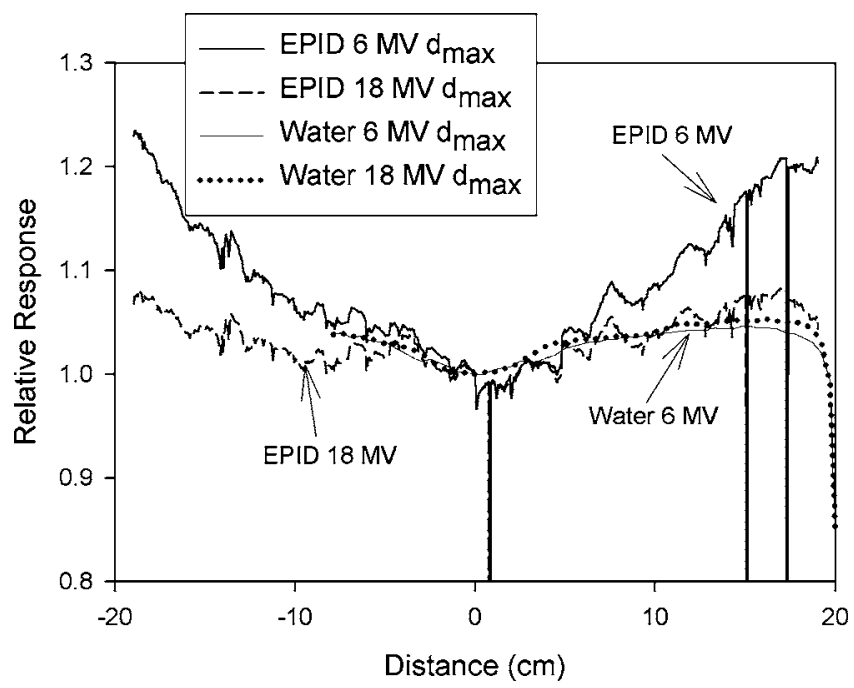

FIG. 2. Comparison of the raw EPID response or flood field with $d_{\max }$ buildup for 6 and $18 \mathrm{MV}$ with $d_{\max }$ dose profiles measured with an ion chamber in water.

energy spectrum. To determine whether this effect is due to the pixel sensitivities, the method was developed to determine these without the effect of beam profile or beam energy variation across the EPID.

\section{B. Pixel sensitivity variation}

Figure 3 shows the measured pixel sensitivity variation of the EPID along the central axis in the cross-plane direction for both 6 and $18 \mathrm{MV}$. The pixel sensitivity variation is relatively small across the EPID compared with the conventional FF sensitivity correction (Fig. 2). The variation is very similar for 6 and $18 \mathrm{MV}$ when measured with each pixel in the detector exposed to virtually the same beam profile and beam

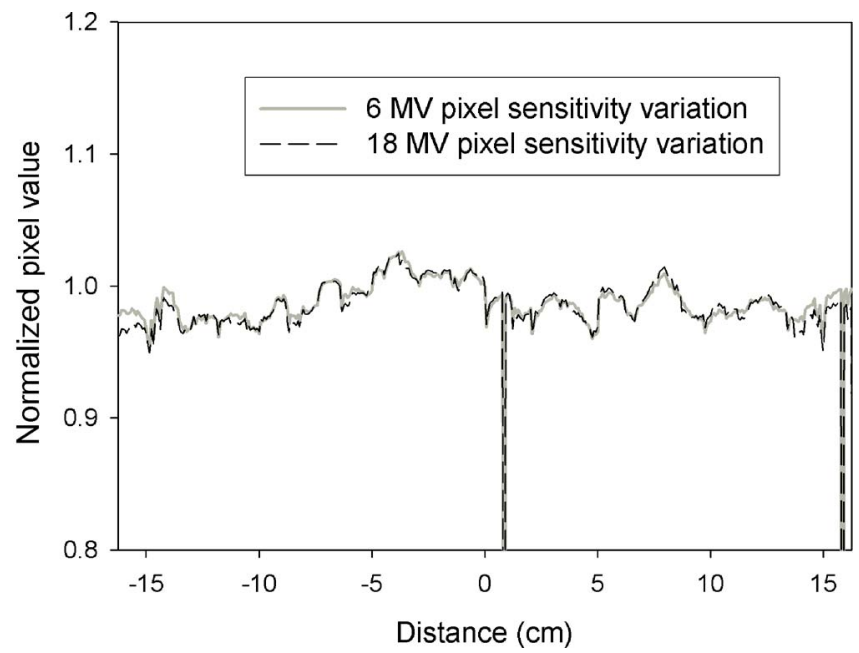

FIG. 3. Pixel sensitivity variation of the EPID measured by combining multiple images of a $10 \mathrm{~cm}$ width field with the detector displaced laterally for each image. The sensitivity variation along the central axis in the crossplane direction is similar for 6 and $18 \mathrm{MV}$ beams.

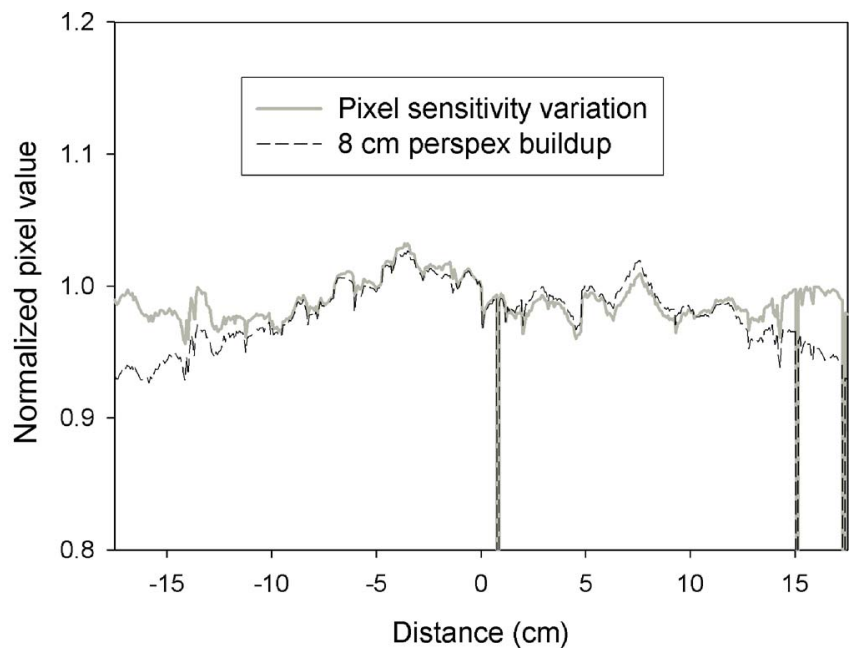

FIG. 4. Pixel sensitivity variation of the EPID for $6 \mathrm{MV}$ photons compared to the conventional FF measured by approximating a large "flat" input beam with $8 \mathrm{~cm}$ of Perspex buildup on the EPID. This method agrees with the pixel sensitivity up to approximately $10 \mathrm{~cm}$ off-axis.

spectrum. The negative spikes in the pixel sensitivity profile are due to defective or "bad" pixels in the matrix, that have markedly different response.

In Fig. 4 the sensitivity variation for $6 \mathrm{MV}$ is shown compared with the conventional FF calibration method made with $8 \mathrm{~cm}$ of Perspex buildup to give a "flat" incident beam to the EPID. This buildup is equivalent to $10 \mathrm{~cm}$ of water, and we have previously verified that the beam is relatively flat at this depth by ion-chamber measurements in water phantom. These are very similar over the central $\pm 10 \mathrm{~cm}$ of the beam. For greater off-axis distances the beam profile at the EPID through the Perspex buildup could be falling off and affecting the measured sensitivities.

Figure 5 illustrates the method to obtain the two-

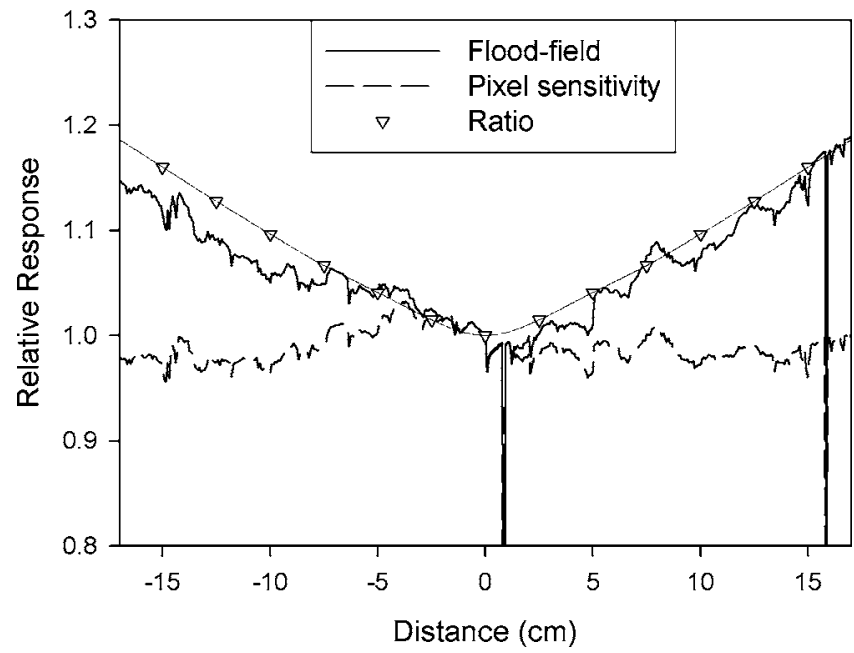

FIG. 5. This figure illustrates how the two-dimensional pixel sensitivity map (PSM) was determined for the $6 \mathrm{MV}$ beam. The ratio between the open FF and the pixel sensitivity variation in the cross-plane direction was determined at $2.5 \mathrm{~cm}$ intervals, and a smooth function fitted to these to form a ratio function. This ratio function was then extended radially and applied to the open FF to obtain the PSM. 


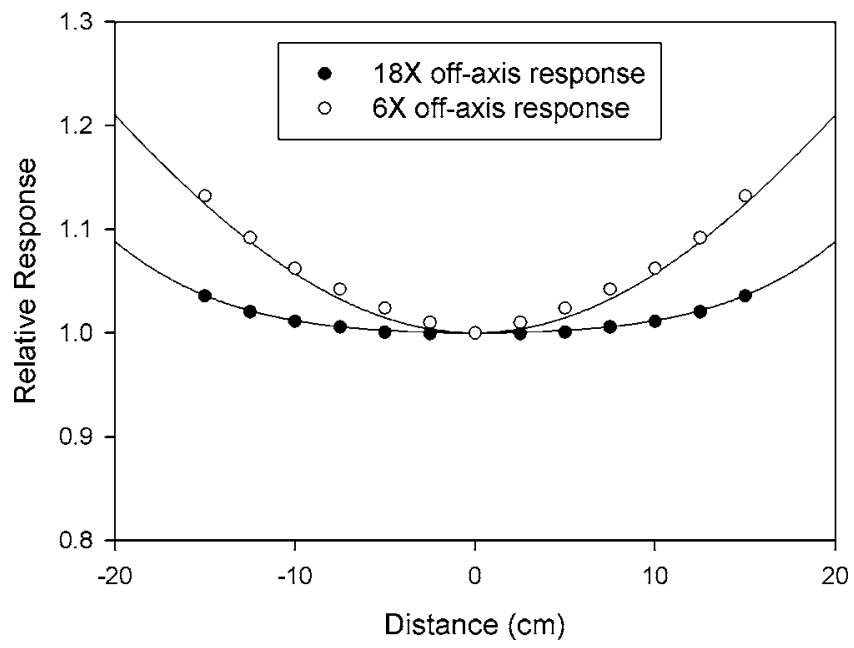

FIG. 6. Off-axis differential energy response of the EPID for 6 and $18 \mathrm{MV}$ photon beams, defined at $100 \mathrm{~cm}$ source to plane distance compared to an ion chamber in water.

dimensional pixel sensitivity map (PSM). The ratio between the conventional open FF cross-plane profile and the pixel sensitivity variation cross-plane profile is shown. This ratio was calculated at $2.5 \mathrm{~cm}$ intervals in the center of the fields used to form the pixel sensitivity variation, and a smooth function fitted to these. This avoided discontinuities in the ratio function. The discontinuities arose at the junctions of the $2.5 \mathrm{~cm}$ segments of the cross-plane pixel sensitivity profile due to asymmetries in the beam profile in the central region of the $10 \times 25 \mathrm{~cm}^{2}$ fields. This ratio function includes the beam profile and off-axis differential response of the EPID with respect to an ion chamber in water in the crossplane direction along the central axis. This ratio function was then extended radially, and mapped to a two-dimensional matrix. The conventional open FF 2D matrix was then divided by this $2 \mathrm{D}$ function to obtain the complete PSM.

The difference between the open-field EPID profile with the bare imager and with $d_{\max }$ buildup was less than $1 \%$ at $15 \mathrm{~cm}$ from the central axis for both energies. Therefore, the ratio function developed here could be utilized to derive the PSM when no added buildup is used on the EPID with reasonable accuracy.

\section{Off-axis response}

The off-axis differential energy response of the EPID is shown in Fig. 6 for both 6 and 18 MV photon energies. The response is defined at a plane $100 \mathrm{~cm}$ from the source. The EPID clearly has a significant off-axis differential response with respect to an ion chamber in water compared to a central axis point. The response at $18 \mathrm{MV}$ is smaller, increasing to $3.5 \%$ at $15 \mathrm{~cm}$ relative to the central axis. However, the response is quite large at $6 \mathrm{MV}$, being $13 \%$ at $15 \mathrm{~cm}$ from the central axis. A fourth-order polynomial $\left(y=p_{1} x^{4}+p_{2} x^{3}\right.$ $\left.+p_{3} x^{2}+p_{4} x+p_{5}\right)$ was fitted to the $6 \mathrm{MV}$ result, with the fitted parameters being $p_{1}=-1.527 \times 10^{-7}, p_{2}=-8.928 \times 10^{-20}, p_{3}$ $=5.86 \times 10^{-4}, p_{4}=1.272 \times 10^{-17}$, and $p_{5}=1.0063$. The values of $p_{2}$ and $p_{4}$ are negligibly small and are not required. The fit

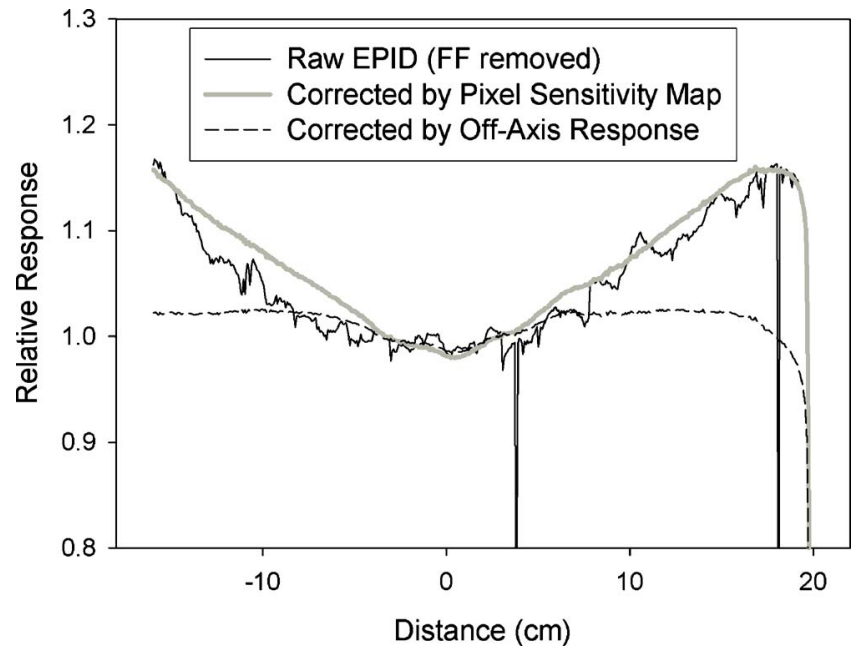

FIG. 7. Illustration of the new correction method for EPID dosimetry. The raw EPID pixel values are obtained by removal of the FF correction. These are then corrected for pixel sensitivity variation by the PSM. The result is corrected for the off-axis differential energy response of the EPID, with this function applied about the beam central axis and scaled to the EPID distance.

was adjusted to $p_{5}=1$ so that the central axis value was 1 . A quadratic function also fitted the data well, although there were small differences not apparent with the polynomial. The $18 \mathrm{MV}$ was similarly fitted with a fourth-order polynomial, with the fitted parameters being $p_{1}=3.418 \times 10^{-7}, p_{2}$ $=-8.77 \times 10^{-20}, p_{3}=8.351 \times 10^{-5}, p_{4}=1.260 \times 10^{-17}$, and $p_{5}$ $=1.000$. This response was measured with an open field of $10 \mathrm{~cm}$ width and $25 \mathrm{~cm}$ length. The beam spectrum off-axis will vary for physical wedges and IMRT fields, and the response correction required may therefore be different.

Whether the off-axis response derived here is applicable to the imager without added buildup was examined. The ratio of the response at the center of an off-axis image recorded at $12.5 \mathrm{~cm}$ off-axis to the central axis response without buildup was compared to the same quantity with the $d_{\max }$ buildup. These ratios were within $0.5 \%$, with the ratio without buildup higher than with buildup for $6 \mathrm{MV}$, and lower for $18 \mathrm{MV}$. Therefore, the off-axis differential energy response derived with this method without added buildup on the EPID would be expected to be within approximately $0.5 \%$ of the results here.

\section{Correction of EPID images}

The process of EPID image correction for dosimetry with the new method is illustrated in Fig. 7. The raw EPID pixel values are obtained by removal of the automatically applied FF correction. This removes any artifacts due to misalignment of the raw EPID image and the FF. The raw pixel values are then corrected for pixel sensitivity variation by the PSM. The result is corrected for the off-axis differential energy response of the EPID, with this function applied about the beam central axis, and scaled to the EPID distance. In this case the EPID was displaced by $3.0 \mathrm{~cm}$ laterally, with a 


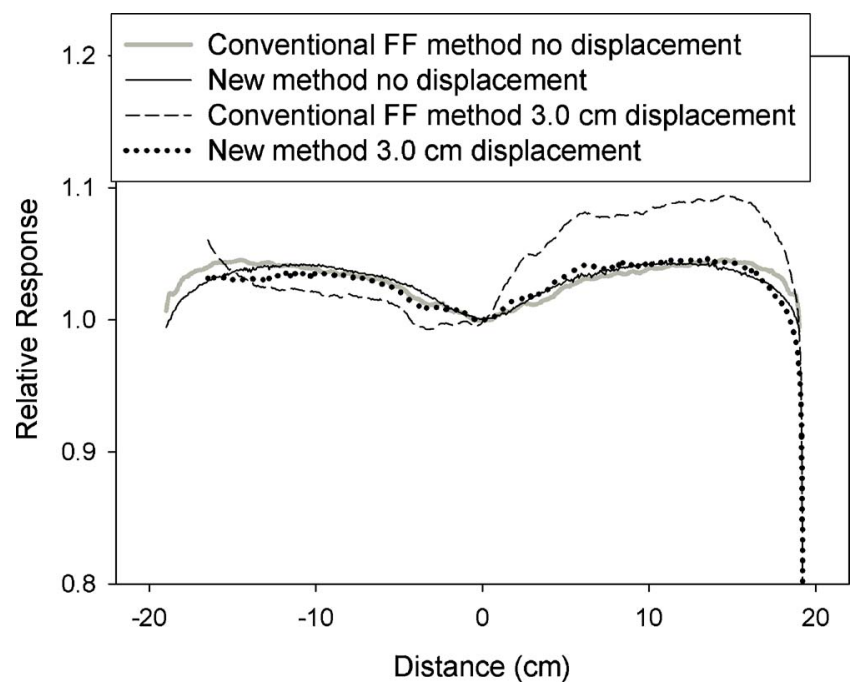

FIG. 8. Effect of lateral detector displacement between FF calibration and subsequent dosimetry measurement for conventional FF calibration and the new method developed here. For the conventional FF method the beam profile has been restored by multiplication by a beam profile. The detector displacement causes dosimetric artifact with this method due to the mismatch of the raw response and the FF.

$40 \times 30 \mathrm{~cm}^{2}$ open field. The FF-corrected EPID image contained significant asymmetry as shown in the following section.

\section{E. Effect of EPID displacement and dosimetry measurements}

The magnitude of the dosimetric error with lateral displacement of the detector was found to increase at the rate of approximately $1 \%$ per $\mathrm{cm}$ at $6 \mathrm{MV}$. This is defined as the percentage differences from the flat profile without the EPID displaced, determined by division of the profiles. The errors were smaller for vertical detector displacement, being $2 \%$ for the $20 \mathrm{~cm}$ vertical displacement, or approximately $0.1 \%$ per $\mathrm{cm}$ of vertical displacement. The new correction method gave agreement between EPID profiles with detector displacement of within $1 \%$ for all displacements. A 20 $\times 20 \mathrm{~cm}^{2}$ field recorded with the EPID displaced $7.5 \mathrm{~cm}$ laterally was also within $1 \%$ of the result for a $20 \times 20 \mathrm{~cm}^{2}$ field with no EPID displacement. This was assessed for within $\pm 9 \mathrm{~cm}$ from the central axis, i.e., excluding penumbra (result not shown). The percentage difference is obtained by dividing the two profiles, and calculating the absolute value of the result.

A comparison of the effect of detector displacement on the dosimetric beam profile for the new method and conventional FF calibration with beam profile correction is shown in Fig. 8. This result is with a detector displacement of $3.0 \mathrm{~cm}$ laterally in the cross-plane direction. The conventional FF calibration image has been multiplied by a $40 \times 40 \mathrm{~cm}^{2} d_{\max }$ water tank profile centered on the beam central axis to "restore" the beam profile to the image. The restored beam profile matches the EPID profile obtained with the new method when no detector displacement is present. However, for the $3.0 \mathrm{~cm}$ detector displacement the dosimetric errors in the

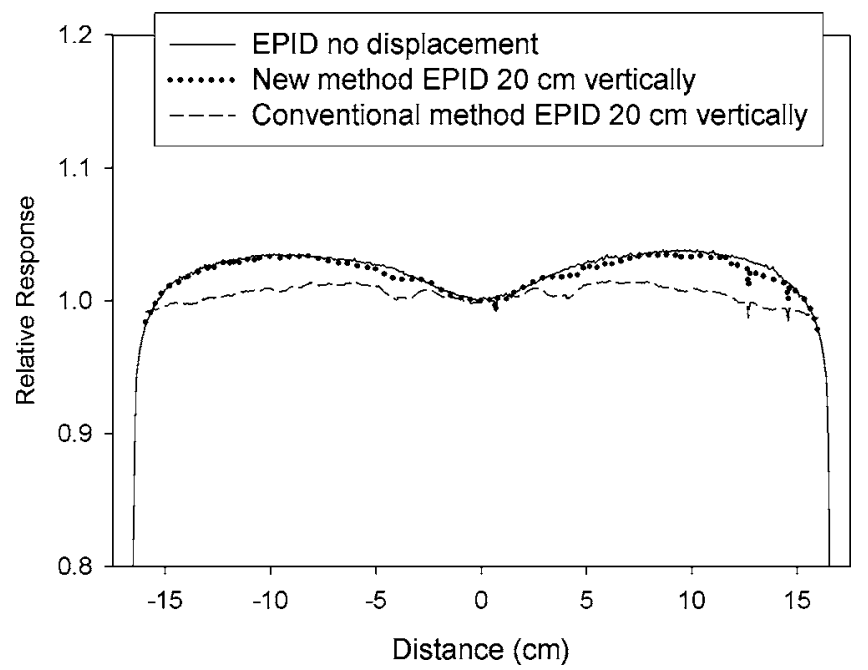

FIG. 9. Effect of vertical detector displacement between FF calibration and subsequent dosimetry measurement. The detector was placed at $105 \mathrm{~cm}$ from the source for FF calibration and $125 \mathrm{~cm}$ from the source for dosimetry measurement. The conventional FF-calibration image was multiplied by the beam profile that was scaled to the detector distance. The mismatch of the raw EPID response and the FF results in a flattening of the recorded profile.

EPID profile with the conventional FF calibration method are large. This is due to the misalignment of the beam profile and off-axis response in the raw EPID response and the FF calibration image. The beam profile is not accurately restored by multiplication of the image by a beam profile function, and the image is not useful for accurate dosimetry. The result for the new method shows agreement between the profiles of within $1 \%$.

Figure 9 shows the effect of the $20 \mathrm{~cm}$ vertical detector displacement between conventional FF calibration and dosimetry measurement. Again, the conventional FF-corrected image has been multiplied by a $40 \times 40 \mathrm{~cm}^{2} d_{\max }$ water tank profile scaled to the detector distance. When the EPID is displaced vertically the slope of the raw EPID profile and FF profile are mismatched due to the change in beam profile, and off-axis differential energy response with the increased distance from the source. This again introduces dosimetry errors due to FF division, with a flattening of the EPID profile. The beam profile again is not restored by multiplication of the EPID image by the beam profile function. However, the new method performs well with vertical detector displacement with only very small differences $(<1 \%)$ between the profiles.

Figure 10 shows the EPID profiles corrected for pixel sensitivity variation and off-axis differential energy response with the new method for a $40 \times 30 \mathrm{~cm}^{2}$ field, compared to $d_{\max }$ ion-chamber measurements of a $40 \times 40 \mathrm{~cm}^{2}$ field in water. Both in-plane and cross-plane directions for 6 and $18 \mathrm{MV}$ are shown. The correction method does not wash out the incident beam profile, and the resulting EPID dose profile agrees well with the $d_{\max }$ dose in water to within $1 \%$ for $6 \mathrm{MV}$ and $1.3 \%$ for $18 \mathrm{MV}$. Figure 10 also shows the comparison of EPID and an ion chamber for open fields of 5 $\times 5,10 \times 10$, and $20 \times 20 \mathrm{~cm}^{2}$ for both 6 and $18 \mathrm{MV}$ in the 

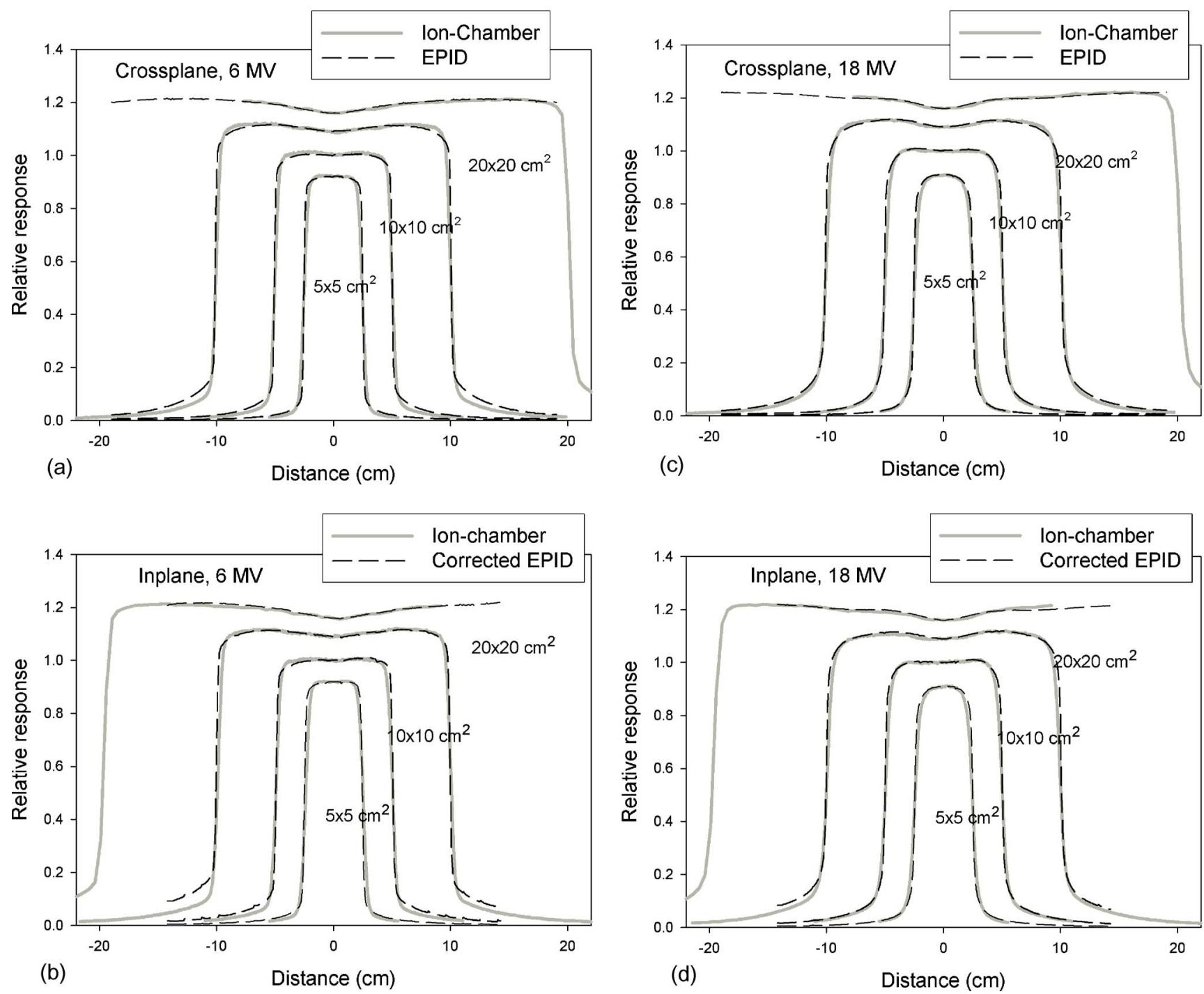

FIG. 10. Comparison of 6 and 18 MV EPID profiles for $5 \times 5,10 \times 10,20 \times 20$, and $40 \times 30 \mathrm{~cm}^{2}$ fields with $d_{\max }$ ion-chamber measurement in water for (a) $6 \mathrm{MV}$ cross plane; (b) $6 \mathrm{MV}$ in plane; (c) $18 \mathrm{MV}$ cross plane; and (d) $18 \mathrm{MV}$ in plane. The profiles have been scaled according to the central-axis pixel values relative to the $10 \times 10 \mathrm{~cm}^{2}$ value. The large-field ion-chamber profile was measured with a $40 \times 40 \mathrm{~cm}^{2}$ field size.

cross-plane and in-plane directions. For display, the profiles have been normalized to the EPID central-axis dose relative to the $10 \times 10 \mathrm{~cm}^{2}$ value. The difference is less than $2 \%$ between the EPID and ion-chamber measurements for the region from central axis to within $1 \mathrm{~cm}$ of the $50 \%$ edge (i.e., excluding the penumbra). This difference was obtained by division of the profiles. The penumbral shape differs due to the difference in scatter kernel of the EPID to water. The penumbra and out-of-field agreement for $18 \mathrm{MV}$ suggest that the scatter kernels of the EPID and water are more similar for $18 \mathrm{MV}$ than they are for $6 \mathrm{MV}$. For IMRT, delivery dose in the penumbral region is important and consideration of the scatter kernel of the EPID is needed. The agreement between corrected EPID and ion-chamber measurements in the inplane direction suggests that the assumption of radial symmetry for the ratio function and the off-axis response is reasonable.

\section{DISCUSSION}

The slope in the raw EPID response seen in Fig. 2 is not caused by a gradient in pixel response or gain over the EPID surface, due for example to a manufacturing process. It is likely that the off-axis differential response of the EPID with respect to an ion chamber in water is due to changes in the beam spectra off-axis, as the response is much higher for $6 \mathrm{MV}$ photons. McCurdy et $\mathrm{al}^{3}{ }^{3}$ found that the EPID overresponds at central axis to low-energy photons due to the photoelectric effect in the high atomic number gadolinium oxysulfide phosphor layer. The mass attenuation coefficient of gadolinium oxysulfide is relatively constant above $1 \mathrm{MeV}$. However, below $1 \mathrm{MeV}$ it rapidly rises to be over 3 orders of magnitude higher at $10 \mathrm{keV}$ than at $1 \mathrm{MeV} .{ }^{13}$ It is well known that the beam energy decreases with off-axis distance. Mohan et al. ${ }^{14}$ found that the mean energy of a 
6 $\mathrm{MV}$ beam decreases from $1.9 \mathrm{MeV}$ on-axis to $1.5 \mathrm{MeV}$ at $15-20 \mathrm{~cm}$ off-axis. The mean energy of a higher energy (15 MV) beam also decreases with off-axis distance; ${ }^{14}$ however, the mean energy is much higher (decreasing from 4.1 to $3.0 \mathrm{MeV}$ ) than that of the $6 \mathrm{MV}$ beam. The component of photons of energy below $1 \mathrm{MeV}$ is higher for $6 \mathrm{MV}$ photons than $15 \mathrm{MV}$. Further work will investigate in more detail the off-axis differential energy response. This differential response off-axis of the EPID with respect to an ion chamber in water is clearly a significant issue for dosimetry, particularly for the lower $6 \mathrm{MV}$ energy. The results for the measurement of the PSM with $8 \mathrm{~cm}$ of Perspex buildup suggests that the spectrum may be sufficiently filtered to largely remove the EPID spectral response; however, this requires further investigation.

The off-axis differential energy response was derived for a $10 \times 25 \mathrm{~cm}$ open field. Applying this correction for all irradiation conditions assumes that the beam spectrum off-axis (and hence EPID response) does not change. This may not be the case for different field sizes, physical wedges, or IMRT fields, where the beam spectrum may be modified by transmission through shielding. Where the EPID is used for in vivo measurements, the spectrum will also be modified by transmission through the patient. The off-axis differential energy response of the EPID will also depend on the amount of buildup material used for dosimetry measurements, as this may affect the relative beam spectrum incident on the imager. Without added buildup on the EPID, the off-axis differential energy response would be within approximately $0.5 \%$ of the results here with $d_{\max }$ buildup. The response would be slightly higher for $6 \mathrm{MV}$ without added buildup on the EPID. This could be due to the fact that the added buildup filters some of the lower energy photons off-axis. However, at $18 \mathrm{MV}$ the off-axis response was slightly lower without buildup.

Another limitation is the assumption of radial symmetry in both the ratio function and the off-axis differential energy response. To calculate the $2 \mathrm{D}$ responses by radially extending the cross-plane ratio function, it was necessary to remove asymmetry in the ratio function. As this asymmetry was actually a property of the beam in the cross-plane direction, then spurious asymmetry is introduced into the PSM (in the cross-plane direction) by removing it. However, this was found to be less than $0.5 \%$. The linear extrapolation of the ratio function was arbitrary. The ratio function will depend on the interplay between the beam profile and the off-axis differential energy response of the EPID, and is therefore difficult to predict. The extrapolated region will only apply to the region outside the field for field widths less than $30 \mathrm{~cm}$ in any dimension.

The raw measured functions contained undulations that were due to the fact that the $10 \times 25 \mathrm{~cm}^{2}$ field used for the measurements was not completely flat, even over the central $\pm 2.5 \mathrm{~cm}$ of the beam that was used. The $18 \mathrm{MV}$ results also showed a "discontinuity" at the matching point of each field segment. This was due to an asymmetry in the central portion of the $18 \mathrm{MV}$ cross-plane beam profile. This was over- come by taking the central value at $2.5 \mathrm{~cm}$ intervals corresponding to the center of each $2.5 \mathrm{~cm}$ segment. Although a smaller sampling interval could have been used with more images acquired, the results for the off-axis differential energy response show that these are slowly varying quantities and that $2.5 \mathrm{~cm}$ was sufficient sampling. It was also necessary to extrapolate the off-axis differential energy response to account for detector displacement. Although this extrapolation is large and will introduce uncertainty into the off-axis response, the extrapolated correction is likely to be in the region of the beam outside the field. This is because the maximum beam dimension in any direction from the central axis is $\pm 20 \mathrm{~cm}$ at isocenter (and most beams are smaller), so the off-axis correction beyond this distance will be outside the field edge. When the detector distance from isocenter is increased, only smaller fields can be imaged without irradiation of the surrounding electronics. A polynomial fit to the measured data was used for the extrapolation. A more accurate extrapolation could potentially be made based on theoretical considerations or Monte Carlo modeling.

An advantage of the conventional FF-calibration method is that the off-axis differential energy response is in both the raw EPID image and the FF-calibration image. Provided the detector position is identical, then the off-axis differential energy response will be corrected (for that irradiation condition). However, the beam profile is also "washed out" and must be restored with an a priori estimate of the profile. ${ }^{7}$ With the method developed here, the pixel sensitivities and off-axis differential energy response corrections do not contain the beam profile, and the beam profile information is retained in the EPID image. The results show that the corrected EPID response is very similar to $d_{\max }$ ion-chamber dose in water, with discrepancies in the penumbra region of the profiles due to the difference in scatter kernel of water and EPID, and the glare kernel of the EPID. ${ }^{8}$

The EPID profiles in the in-plane direction for the smaller field sizes show a slope in the profiles compared to water tank profiles, with the dose reducing in the direction towards the gantry. Siebers et al. ${ }^{11}$ found that backscatter from the EPID mounting bracket that sits under the EPID side closest to the gantry affects the EPID signal in this area. While backscatter would be expected to increase the dose, the reason that the profiles decrease lies in the derivation of the PSM. This was derived from the open FF image acquired under maximum backscatter conditions. Therefore, the PSM contains the maximal backscatter to each pixel. When a smaller field size is used, the backscatter to the pixel will be reduced, as less of the mounting bracket is exposed to the beam. Therefore, the PSM correction lowers the EPID signal to the pixel by overestimating the backscatter component. This effect will decrease with increasing distance from the mounting bracket giving the slope to the profiles. Ko et al. ${ }^{9}$ have suggested that a layer of lead be placed between the EPID and the mounting to reduce this backscatter.

In this work, an off-axis differential energy response with respect to an ion chamber in water for the aS500 EPID is quantified. In conventional FF calibration, the pixel sensitivity variation and off-axis differential energy response are 
combined in one correction matrix. As the sensitivity of a pixel does not depend on the detector location, while the off-axis differential energy response depends on the distance of the pixel from central axis, this does not allow correction of these quantities for detector displacement from the FFcalibration reference position. For IMRT verification with conventional FF calibration, where it may be desirable to use the actual gantry angles of the fields, the EPID position should be corrected for any detector sag that introduces lateral and longitudinal detector displacement at each gantry angle. This can be achieved using a marker on the EPID to align with the central-axis optical crosshair. Alternatively, where the EPID image is compared with a theoretical prediction, the off-axis differential energy response could be incorporated into the predicted image. This would be a simpler approach, as it would eliminate the need to account for or correct the EPID position, provided the EPID image is registered to the prediction.

To implement this method in the clinic will require that the FF be measured with sufficient buildup to obtain a flat beam without significant energy response, or calculated from the ratio function between open FF and PSM. That the function determined here applies to the local EPID and beam should be verified. It was not found possible to fit an analytical expression to this ratio function. A spline fit to the points in Fig. 5 was made, followed by linear extrapolation. Similarly the off-axis differential energy response may be similar for different aS500 EPIDs for the same beam energies employed here. If this is the case then the analytical function can be used to produce the off-axis differential energy response correction.

\section{CONCLUSIONS}

The conventional FF pixel sensitivity correction method for EPID dosimetry removes beam profile information from the image. Moreover, if the detector is displaced from the FF-calibration position, then dosimetric errors occur due to misalignment of the beam profile and off-axis differential energy response in the raw EPID image and FF-correction image. A method has been developed that explicitly determines the pixel sensitivity function and the off-axis differential energy response of the EPID. This allows correction of EPID images for these at any detector position. The results here show that the EPID dosimetry measurements are then virtually independent of detector position, and the beam profile is directly measured. The beam penumbra measurements differ for the EPID and ion chamber, and these differences may be important in applications such as IMRT dosimetry. The amorphous silicon EPID was found to have a large offaxis differential energy response with respect to an ion chamber in water, particularly for $6 \mathrm{MV}$ that should be considered for EPID dosimetry.

\footnotetext{
${ }^{a)}$ Electronic mail: peter.greer@mater.health.nsw.gov.au

${ }^{1}$ P. Munro and D. C. Bouius, "X-ray quantum limited portal imaging using amorphous silicon flat-panel arrays," Med. Phys. 25, 689-702 (1998).

${ }^{2}$ Y. El-Mohri, L. E. Antonuk, J. Yorkston, K. W. Jee, M. Maolinbay, K. L. Lam, and J. H. Siewerdsen, "Relative dosimetry using active matrix flatpanel imager (AMFPI) technology," Med. Phys. 26, 1530-1541 (1999). ${ }^{3}$ B. M. C. McCurdy, K. B. Luchka, and S. Pistorius, "Dosimetric investigation and portal dose image prediction using an amorphous silicon electronic portal imaging device," Med. Phys. 28, 911-924 (2001).

${ }^{4}$ P. B. Greer and C. C. Popescu, "Dosimetric properties of an amorphous silicon electronic portal imaging device for verification of dynamic intensity modulated radiation therapy," Med. Phys. 30, 1618-1627 (2003).

${ }^{5}$ J. Chang and C. C. Ling, "Using the frame-averaging of aS500 EPID for IMRT verification,” J. Appl. Clin. Med. Phys. 4, 287-299 (2003).

${ }^{6}$ A. van Esch, T. Depuydt, and D. P. Huyskens, "The use of an aSi-based EPID for routine absolute dosimetric per-treatment verification of dynamic IMRT fields," Radiother. Oncol. 71, 223-234 (2004).

${ }^{7}$ B. Warkentin, S. Steciw, S. Rathee, and B. G. Fallone, "Dosimetric IMRT verification with a flat-panel EPID," Med. Phys. 30, 3143-3155 (2003).

${ }^{8}$ C. Kirkby and R. Sloboda, "Comprehensive Monte Carlo calculation of the point spread function for a commercial a-Si EPID," Med. Phys. 32, 1115-1127 (2005).

${ }^{9}$ L. Ko, J. O. Kim, and J. V. Siebers, "Investigation of the optimal backscatter for an aSi electronic portal imaging device," Phys. Med. Biol. 49, 1723-1738 (2004).

${ }^{10}$ J. V. Siebers, J. O. Kim, P. J. Keall, and R. Mohan, "Pre-treatment verification of IMRT fields using measured and Monte Carlo computed dose images," Presented at the 7th International Workshop on Electronic Portal Imaging, Vancouver, BC, Canada, 2002.

${ }^{11}$ J. V. Siebers, J. O. Kim, L. Ko, P. J. Keall, and R. Mohan, "Monte-Carlo computation of dosimetric amorphous silicon electronic portal images," Med. Phys. 31, 2135-2146 (2004).

${ }^{12}$ H. Parsaei, E. El-Khatib, and R. Rajapakshe, "The use of an electronic portal imaging system to measure portal dose and portal dose profiles," Med. Phys. 25, 1903-1909 (1998).

${ }^{13}$ National Institute of Standards and Technology, Physical Reference Data, Tables of X-Ray Mass Attenuation Coefficients and Mass EnergyAbsorption Coefficients.

${ }^{14}$ R. Mohan, C. Chui, and L. Lidofsky, "Energy and angular distributions of photons from medical linear accelerators," Med. Phys. 12, 592-597 (1985).
} 